\title{
Dynamic cognitive characteristics of emotional conflict in subthreshold depressed patients: An Event-Related Potential Study
}

\author{
Weizhou Wang \\ Shandong University of Chinese Medicine \\ Qi Xie \\ Shandong University of Chinese Medicine \\ Lulu Zhang \\ Shandong University of Chinese Medicine \\ Shuang Hu ( $\sim 1058791076 @ q q . c o m$ ) \\ Shandong University of Chinese Medicine
}

Research Article

Keywords: subthreshold depression (SubD), emotional conflict, N2, N450, conflict SP

Posted Date: December 8th, 2021

DOI: https://doi.org/10.21203/rs.3.rs-1103862/v1

License: (c) (i) This work is licensed under a Creative Commons Attribution 4.0 International License. Read Full License 


\section{Abstract}

Subthreshold depression (SubD) has a considerable impact on an individual's subjective well-being and psychosocial functioning, and is a risk factor for Major depression disorder (MDD). The inability to effectively control and resolve emotional conflict is a typical symptom of certain mood disorders, and the aim of this study was to confirm impairments in cognitive processing mechanisms for emotional conflict processing in SubD patients with event-related potential (ERP) recording. The study of the mechanisms of emotional conflict in subthreshold depression may provide an ideal model for understanding the neurophysiological mechanisms and developing preventive strategies in patients with MDD.

Methods:The Healthy control (HC) and SubD groups were recruited, with 32 subjects in each group completing the word-face Stroop paradigm, during which ERP amplitudes and latencies were recorded.

Results:Compared to HC group, the SubD group had lower accuracy and longer response times in both the "consistent stimulus" and "inconsistent stimulus" conditions. Regardless of the stimulus condition, the SubD group had a greater N2 amplitude in the prefrontal mid-lobe region. In the SubD group, the N450 amplitude was also found to be greater in the prefrontal middle region for the "incongruent stimulus minus congruent stimulus" and the conflict SP amplitude was smaller in the parieto-occipital region for the "incongruent stimulus minus congruent stimulus".

Conclusions: The findings suggest that, supported by behavioural and brain evidence, people with SubD have dynamic cognitive deficits in emotional conflict processing, specifically greater sensitivity to early processing of emotional stimuli and sharper detection of emotional conflict, but more delayed adaptation and response options following emotional conflict resolution.

\section{Introduction}

Major depression disorder (MDD) has a high rate of disability and death and has become a major cause of worldwide burden of disease (Ferrari et al., 2013). It is critical to determining the factors that contribute to the increased risk of MDD. Patients with subthreshold depression (SubD) have the similar symptoms as those with depression, but to a lesser degree and do not meet the criteria for a diagnosis of depression (Dai Q et al., 2012; Hayasaka Y et al., 2015). Because of its high prevalence, insidious symptoms (Lee, Y. et al., 2019), and significant negative impact on patients' quality of life (Tuithof, M. et al., 2018), SubD is increasingly becoming a greater burden on health services than MDD. Adolescents are a vulnerable population for SubD (Bertha and Balazs, 2013). Research shows that SubD affected 32\% of Chinese university students (Jiang et al., 2019) and $23 \%-39 \%$ of European college students (Mikolajczyk et al., 2008). SubD is a risk factor for depression (Bertha and Bala zs,2013). SubD are more likely to develop depression than normal people (Cuijpers and Smit, 2004). A longitudinal study showed the risk of developing SubD into MDD was as high as 10-25\% within 1-3 years compared to the normal population (Cuijpers and Smit, 2004). Therefore, SubD research may provide a superb model for Comprehension the pathophysiology of MDD and further for developing preventive strategies for MDD.

MDD results from abnormalities in emotional processing mechanisms within the brain and manifests as deficits in emotional neurocognitive processes (Disner SG et al., 2011). Specifically, MDD selectively perceives and evaluates the emotional information around it (Disner SG et al., 2011; Everaert J et al., 2012), triggering abnormally high levels of negative emotions and abnormally low levels of positive emotions (Jones, E. B. and Sharpe, L., 2017). Besides this, There is research evidence that MDD suffers from conflict monitoring dysregulation, which is reflected in competitive tasks regarding response options.(Ottowitz WE et al., 2002; Epp AM et al., 2012). This suggests that dysregulated conflict monitoring may be a marker of executive dysfunction in people with MDD. However, conflict monitoring in life is not only cognitive, many emotionally conflicting stimuli in the environment can also interfere with our ongoing behaviors (LeDoux JE, 2000; Tipples J and Sharma D, 2000).

Emotional conflict develops from cognitive conflict and is the interference of unrelated stimuli with the current cognitive task (Etkin A et al., 2006

). Research suggests that the inability to resolve and adapt to emotional conflicts is typical of people with certain mood disorders, such as depression and anxiety disorders (Williams JM, 1996; Xue S, 2017). The study found that compared to healthy controls, people with depression had longer reaction times and lower accuracy in naming the colours of sad words in an emotional stroop paradigm (Ottowitz WE et al., 2002). Therefore, defective emotional conflict processes are an underlying deficit in Depressed people (Anderson ND et al., 2007), and controlling emotional conflict is important for both normal functional performance and treatment of mood disorders. The research on emotional conflict in depressed patients is comprehensive and in-depth, both at the neurological and behavioural mechanism levels (Epp AM et al., 2012), but relatively little research has been done on SubD patients. Research on emotional conflict in patients with SubD is important not only for uncovering the mechanisms of emotional conflict, but also for diagnosis and treatment.

Most previous studies examining emotional conflict have used the emotional stroop paradigm ,but there is no real emotional conflict formed between the emotional meaning of words and the names of colors in this paradigm (Algom D et al., 2004). The word-face Stroop paradigm, which is transformed from the emotional Stroop paradigm, is one of the main paradigms for studying emotional conflict and can directly assess emotional conflict. It operates by marking emotional words in emotionally colored faces and asking subjects to judge the emotional value of the 
face or word (Stenberg G et al.,1998; Kavcic V and Clarke J M, 2000). Previous studies have shown that the event-related potential (ERP) technique is used widely. It is a technique with high temporal resolution that compensates for the shortcomings of other methods in cognitive neuroscience and allows us to better understand cognitive processes.

A series of ERP studies using the word-facet Stroop paradigm found that word-facet incongruent stimuli triggered a more negative N2 component around 200-300ms post-presentation, and that N2 amplitude in the frontal central region may be related to emotion control and conflict detection (Thomas S J et al., 2007; van Hooff J C et al., 2008). In addition, word-face incongruent stimuli triggered a more negative N450 component in the central frontal region of the midline $300-500$ ms after presentation, which is thought to show conflict detection in the Stroop task (Coderre, Conklin and van Heuven, 2011). The wave amplitude was greatest in the left of midline region for N450 in the early phase (350$450 \mathrm{~ms}$ ) and in the central midline region for N450 in the late phase (450-500ms), because early N450 may come from a left frontal location, unlike early N450, late N450 originate from the central frontal and ACC. this suggests that early and late components of N450 may be involved in different stages of cognitive control, respectively (Taake I et al., 2009; Wei D et al., 2010). It has also been found that the conflict slow potential (SP) is a conflict-sensitive positive component over parietal areas during the 700-800ms time window, and we usually associate the SP with conflict resolution (N. DePisapia and T.S. Braver, 2006), response selection (A.T. Chen et al., 2011) and monitoring (M.J. Larson et al., 2009).

Synthesizing existing research, we decided to use erp technology and adopt a word-faced stroop paradigm to study mechanisms of dynamic cognitive processes in patients with SubD during an emotional conflict task. In terms of behavioural outcomes, we assumed the SubD group would have longer reaction time (RT) and lower accuracy (ACC) in the task compared to the HC group. At the neurological level, we hypothesize that SubD patients exhibit abnormal neurophysiological indicators, such as an overall increase in N2, N450 and SP amplitude levels in the wordface dysregulation condition, where SubD patients exhibit interference effects. This suggests that impaired neural processes underlie the cognitive control deficits associated with SubD.

\section{Materials And Methods}

\section{Participants}

64 undergraduate students (42 women, 22 men) were recruited from the Shandong University of traditional Chinese medicine $\$ Jinan $\llbracket C h i n a \rrbracket$ which through advertisements and grouped according to their scores on the Center for Epidemiological Survey. Depression Scale (CESD) (Radloff LS, 1977)and the Hamilton Depression Scale-17 (Hamilton M, 1967). Both scales had Chinese adaptations and had been widely used. The SubD group were screened using a cutoff score of $\geq 16$ on the CES-D according to previously reported literature (Buntrock $C$ et al., 2017), which is the usual way of indicating "having depressive symptoms" in CES-D. The Hamilton Depression Scale-17 was then used to confirm SD and exclude clinical depression using a score range of between 7 and 17 indicating a mild depression (0 6冈no depression, 7 17囚 mild depression $₫ 18 \sim 24 \rrbracket$ moderate depression $\rrbracket$ above 25 2 severe depression) (Ballesteros J et al., 2007). The Hamilton Depression Scale-17 was performed by at least two assessors, at least one of whom was qualified as a national psychological consultant. All assessors had received training in the assessment's administration. The inclusion criteria for the HC group were CES-D score of $₫ 16$. Exclusion Criteria for both SubD and HC participants were (1) did not fulfill the SCID diagnostic criteria for MDD; (2) had no current bipolar disorder, panic disorder or schizophrenia; (3) had no concurrent psychotherapy and psychotropic medication; and (4)not pregnant and currently not in their menstrual period.

All participants were right-handed, and at the time of the experiment, the SubD group was not receiving medication or was entering the experiment 1 month after weaning from medication. Prior to participating in the experiment, subjects were carefully informed of the purpose of the experiment and the procedure and signed an informed consent form. The study was conducted in accordance with the Declaration of Helsinki as revised in 1989. The subjects were compensated after completing all study procedures.

\section{Experimental material}

The stimulus materials comprised both happy-face and sad-face photographs of people. All photographs were selected from the Native Chinese Affective Picture System (Lu B et al., 2005), which has been deemed to be appropriate for use with the Chinese population. We selected 20 happy-face and 20 sad-face photographs according to a 1:1 sex ratio and used red font for the words " $\mathbb{}$ (' gaoxing' means happy)" or " $\square[$ ('beishang' means sad)" over the central portion of the faces in the photographs. The text appears on the nose area of the face and the font size is approximately $1 \mathrm{~cm} \times 1 \mathrm{~cm}$, an example of which is shown in Figure 1. In the task, there are two conditions (congruent, incongruent), congruent in the sense that the facial expression in the picture agrees with the meaning of the word, and incongruent in the sense that the facial expression does not agree with the meaning of the word The image was eventually rendered on a 17 inch CRT computer screen, measuring $5 \mathrm{~cm}$ by $7 \mathrm{~cm}$.

\section{Experimental Paradigm}

The word-face Stroop paradigm was used in this study. For the specific experimental design, refer to the study by Song Xue et al (Xue, S et al., 2017). The entire experiment comprised one practical trial and four blocks, with each block consisting of 80 trials. After completing a block, the participants were provided 1-2 minutes of rest. When the experiment started, one photograph containing an emotional face and an emotional 
word was shown in the middle of the screen for $1500 \mathrm{~ms}$. The stimulus time interval was between $3000 \mathrm{~ms}$ and $5000 \mathrm{~ms}$ (average: $4000 \mathrm{~ms}$ ), and the central screen displayed a "+" between the stimuli.

For the congruent and incongruent tasks in this study, the experimenters asked the participants to determine whether the face was sad or happy and to press a button in response as soon as possible. The participants were instructed to press the " $\mathrm{S}$ " button with their left-hand finger when the face appeared sad and the " $\mathrm{K}$ " button with their right-hand finger when the face appeared happy. The order of the stimuli was pseudorandom, and all experimental stimuli, as well as the sex of the faces, were balanced. The picture presentation was ended once the participant pressed the correct button or after $1500 \mathrm{~ms}$ had elapsed. A blank screen was then presented for 800 to $1200 \mathrm{~ms}$. In total $\otimes 160$ trials were recorded for each type of stimuli. The whole experiment took about 30 minutes.

The experiment was conducted in a quiet environment, using two computers, one for presenting the stimuli and one for recording the EEG data. During the experiment, subjects were asked to minimise body movements, especially head and facial movements. The subjects were asked to look at the screen with their eyes, their faces $75 \mathrm{~cm}$ away from the screen and their horizontal and vertical viewing angles less than 68 degrees. In order to ensure that the subjects were sufficiently familiar with the whole procedure, a 20-trial pretest was conducted before the formal start of the experiment, and the pre-test procedure was the same as in the formal experiment.

\section{EEG Recording}

In this experiment, a 64-channel electrode cap is used to continuously record EEG. The electrode cap is distributed according to the international $10 / 20$ system, and the sampling rate is $512 \mathrm{~Hz}$. After the original signal is amplified, a bandpass filter $(0.01-100 \mathrm{~Hz})$ is applied. EEG data were collected using a Biosemi (Biosemi, UvA, NL) ActiveTwo Data Conversion Box. Impedances were kept below $5 \mathrm{k} \Omega$ throughout the recording. The preprocessing and analysis of the EEG data was performed in Matlab (version R2014a; MathWorks, Inc, MA, USA) using the EEGlab toolbox (Delorme and Makeig, 2004), the ERPlab toolbox (Lopez-Calderon and Luck, 2014).

The electrodes were referenced to the CMS (Figure 2.) during acquisition and the REST was used as the offline reference, with the study shows that it is more appropriate to use REST reference scheme settings in facial recognition experiments (Wang, Y et al., 2019). Electrodes were placed above and below the left eye and at the outside of the left and the right canthi to measure the electrooculogram. Ocular artefacts were corrected using the independent component analysis (ICA) procedure in the EEGlab toolbox (Delorme and Makeig,2004). The offline high-pass filter is set to 0.1 and the low-pass filter is set to $35 \mathrm{~Hz}$, with a band-pass filter to improve the signal-to-noise ratio. The data 200 ms before the start of the stimulus is used for the baseline correction of the ERP. The continuous EEG is divided into the period starting -200 ms before the start of the stimulus and ending at $1000 \mathrm{~ms}$ after the start of the stimulus. Tests exceeding $\pm 100 \mu \mathrm{V}$ are excluded from further analysis. Epochs containing artefacts were rejected prior to averaging. The average number of accepted trials was $145(S D=6)$ in the word-face emotion consistency condition, $151(\mathrm{SD}=4)$ in the word-face emotion inconsistency condition.

To quantify the ERP data, we calculated mean amplitudes for each condition in 150 250ms, 350 450ms and 700 800ms time windows. We chose the above time window based on previous studies and visual inspection of ERP waveforms. We included the 150 250ms time window to capture the N2 effect, the 350 450ms to capture the N450 effect, and the 700 800ms time window to capture the SP effect. Based on previous research and observation of the difference wave topography. In this study, N2 and N450 in the central prefrontal region and SP in the posterior central region of the parieto-occipital lobe were selected for analysis. Three electrode sites, FZ, FPZ and FCZ, were selected for analysis with average wave amplitudes of N2 and N450 in the central prefrontal region, and three electrode sites, PZ, POZ and OZ, were selected for analysis with average wave amplitudes of SP in the posterior central parieto-occipital region.Please see Figure 3 for details.

\section{Statistical analysis}

Descriptive data were presented as mean $\pm S D$ unless otherwise specified. T-test and $X^{2}$ test were used to compare the demographic data and depression scales (CES-D and Hamilton Depression Scale-17) between SubD participants and HCs.

The E-prime procedure was used to record subject reaction time (RT)of correctly and the accuracy of responses (ACC) spss 22 to analyse these behavioural data. A two factor repeated measure ANOVA with RT and ACC as dependent variables, respectively, was used to examine the group (SubD vs HC) as the between-subject factor, and word-face emotional coherence (congruent vs incongruent) as the within-subject factor. The significance level was 0.05 .

A three-factor repeated measure ANOVA was used to examine the latencies and amplitudes of N2, N450 and SP, with the group (SubD vs HC) as the between-subject factor, and word-face consistency (congruent vs incongruent) xelectrode point (N2 (FZ, FPZ, FCZ); N450冈FZ, FPZ, FCZヌ;SP $\triangle P Z, P O Z, O Z)$ as the within-subject factor. The normality of data was checked before applying parametric tests. The Greenhouse-Geisser correction was used to correct repeated measure ANOVA that violated the sphericity assumption, and the Bonferroni correction was applied to all post hoc pairwise comparisons. The significance level was 0.05 .

Page $4 / 15$ 


\section{Results}

\section{Demographic Characteristics}

Demographic data for subjects in the HC group and SubD group are shown in Table 1 . The mean age was $19.813 \pm 0.859$ years in the SubD group and $19.563 \pm 0.759$ years in the $\mathrm{HC}$ group. The two groups were no statistically significant differences on the age( $\mathrm{t}=-1.233$; $\mathrm{P}=0.222)$, gender $\left(X^{2}=0.549 ; P=0.443\right)$ and education $(t=0.151 ; P=0.880)$ dimensions, showing that the two groups were well matched. In contrast, as an important difference in our setting, CES-D scores are significantly different between HC group.and SubD group ( $t=22.504 ; \mathrm{P} \bigotimes 0.001$ ).

TABLE 1. Group Characteristics (Mean \pm SD $\square$

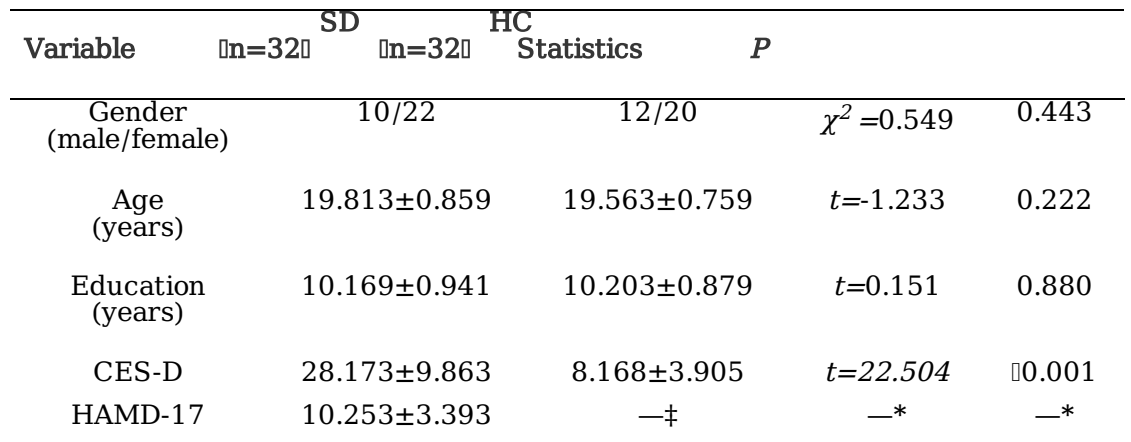

¥: The HAMD-17 was not administered in the HC after screening with the CES-D. *: Group comparisons are not required.

HAMD-17: Hamilton Depression Scale-17; HC, healthy control.

\section{Analysis of Behavioral Data}

The mean and SD of RT and ACC for each condition are shown in Table 2. First, RT was used as a dependent variable to analyze the repeated measures of ANOVA for the group (SubD vs HC) $\times$ word-face emotion consistency (congruent vs incongruent). The results showed that the main effect of group was significant $(F(1,31)=22.361$, $P<0.001)$, and the main effect of word-face emotion consistency was significant $(F(1,31)$ $=55.346, P<0.001)$, and the interaction between them was significant $(F(1,31)=25.432, P<0.001) \otimes$ further simple analysis of the effect shows that the SubD group's RT under the condition of word-face emotion inconsistency was significantly greater than the word-face emotion consistency $(\mathrm{F}(1,31)=8.242, \mathrm{P}<0.001)$, and the HC group's RT under the condition of emotional inconsistency is also significantly greater than the word-face emotional agreement $F(1,31)=3.312, P<0.001)$.

Then, ACC was used as a dependent variable to analyze the repeated measures of ANOVA for the group (SubD vs HC $\mathbb{x} \times$ word-face emotion consistency (congruent vs incongruent). The results showed that the main effect of group was significant $(F(1,31)=5.690, p<0.001)$, and the main effect of word-face emotion consistency was significant $(F(1,31)=96.916, p<0.001)$, other effects were not significant. After pair-wise comparison, it was found that the ACC of SubD group was lower than that of HC group, and under consistent was higher than that under inconsistent.

TABLE 2. Behavioral data comparison between SD and HC (Mean \pm SD)

\begin{tabular}{cccc}
\hline Task & Group & Mean RTs (ms) & Mean ACC \\
\hline Congruent & SubD & $747.093 \pm 16.122$ & $0.901 \pm 0.016$ \\
Incongruent & HC & $659.269 \pm 8.658$ & $0.950 \pm 0.008$ \\
& SubD & $797.560 \pm 20.305$ & $0.812 \pm 0.022$ \\
& HC & $752.149 \pm 13.865$ & $0.869 \pm 0.014$
\end{tabular}

Abbreviations: SubD, Subthreshold depression; HC, Healthy control; RT, response time; ACC, accuracy score. 


\section{ERP Results}

\section{N2}

The latencies and amplitudes of N2 were statistically analyzed. Please see Table 3 and Figure 4 for details. The ANOVA on N2 amplitudes showed a main effect of electrode site (FPZ=-0.609 $\mu \mathrm{V} ; \mathrm{FZ}=-2.787 \mu \mathrm{V} ; \mathrm{FCZ}=-0.803 \mu \mathrm{V} ; \mathrm{F}(2,62)=39.227$, $\mathrm{P}<0.001)$ with the largest amplitudes in the $F Z$ and the smallest amplitudes in the FPZ. There was a main effect of group $(H C=-0.455 \mu V ; S u b D=-2.344 \mu V ; F(1,31)=5.819, P=0.022<0.05) \otimes$ with smaller amplitudes in the HC group than in the SubD group. Between-group comparisons on N2 amplitudes showed the SubD group had smaller amplitudes than the HC group in each type of word-face emotion consistency stimuli. (congruent $\varangle \mathrm{P}=0.047 \otimes 0.05 \rrbracket i n c o n g r u e n t \varangle \mathrm{P}=0.012 \rrbracket$ 0.05). There was no interaction between group and word-face consistency $(F(1,31)=3.227, P=0.082)$.

The ANOVA on N2 latencies showed a main effect of electrode site (FPZ=220.276ms; $F Z=220.429 \mathrm{~ms} ; F C Z=217.240 \mathrm{~ms} ; F(2,62)=4.221$, $\mathrm{P}=0.021<0.05$ ), with smaller latencies in the $\mathrm{FCZ}$ compared with both the $\mathrm{FPZ}$ and the $\mathrm{FZ}$. There was no main effect of group (HC=219.523ms $\mathbb{B}$ SubD=219.106ms; $F(1,31)=0.074, P=0.787)$. No interaction between group and word-face consistency $(F(2,62)=0.707, P=0.497)$.

\begin{tabular}{|c|c|c|c|c|c|}
\hline Task & Group & & FZ & FPZ & FCZ \\
\hline \multirow[t]{4}{*}{ Congruent } & SD & Amplitude & $-3.567 \pm 0.567$ & $-1.532 \pm 0.540$ & $-1.732 \pm 0.459$ \\
\hline & & Latency & $218.567 \pm 2.958$ & $220.520 \pm 3.145$ & $219.788 \pm 3.294$ \\
\hline & $\mathrm{HC}$ & Amplitude & $-2.107 \pm 0.638$ & $0.213 \pm 0.724$ & $-0.001 \pm .756$ \\
\hline & & Latency & $223.145 \pm 1.933$ & $221.436 \pm 2.829$ & $216.675 \pm 3.378$ \\
\hline \multirow[t]{4}{*}{ Incongruent } & SD & Amplitude & $-3.691 \pm 0.548$ & $-1.702 \pm 0.475$ & $-1.839 \pm 0.426$ \\
\hline & & Latency & $219.422 \pm 2.971$ & $220.032 \pm 3.181$ & $216.309 \pm 3.516$ \\
\hline & $\mathrm{HC}$ & Amplitude & $-1.781 \pm 0.623$ & $0.585 \pm 0.790$ & $0.362 \pm 0.781$ \\
\hline & & Latency & $220.581 \pm 2.584$ & $219.116 \pm 3.277$ & $216.187 \pm 3.422$ \\
\hline
\end{tabular}

\section{N450}

The amplitudes and latencies of N450 were statistically analyzed. Please see Table 4 and Figure 4 for details. The ANOVA on N450 amplitudes showed a main effect of electrode site $(F Z=-1.511 \mu \mathrm{V} ; F C Z=0.350 \mu \mathrm{V} ; F P Z=0.219 \mu \mathrm{V} ; \mathrm{F}(2,62)=21.783, \mathrm{P}<0.001$ with the largest amplitudes in the $F Z$ and the smallest amplitudes in the FCZ. There was a main effect of group $(H C=0.694 \mu V \Downarrow S u b D=-1.322 \mu V ; F(1,31)=8.136, P=0.008<0.05) \rrbracket$ with smaller amplitudes in the $\mathrm{HC}$ group than in the SubD group. There was a main effect of word-face emotion consistency (congruent $=-0.112 \mu \mathrm{V}$; incongruent $=-0.517 \mu \mathrm{V} ; \mathrm{F}(1,31)=20.615, \mathrm{P}<0.001$ ), and it was found that there was a trend interaction between group and word-face emotion consistency $(F(1,31)=5.051, P=0.032<0.05)$, further analysis of the simple effect showed that the HC group had a smaller differential wave of $\mathrm{N} 450$ induced by discordant and consistent conditions than the SubD group (differential wave of HC group=-0.210 $\mu \mathrm{V}$; differential wave of SubD group $=-0.600 \mu \mathrm{V} ; \mathrm{P}=0.001 \otimes 0.001)$.

The ANOVA on N450 latencies showed a main effect of group $(H C=409.638 m s ; S D=403.107 m s ; F(1,31)=6.799 ; P=0.014 \bowtie 0.05)$, with longer latencies in the SubD group than in the HC group. There was no main effect of word-face emotion consistency (congruent=406.281ms; incongruent=406.464ms; $F(1,31)=0.016, P=0.899)$. No interaction between group and word-face consistency $(F(1,31)=1.818, P=0.187)$. 


\begin{tabular}{|c|c|c|c|c|c|}
\hline Task & Group & & FZ & FPZ & FCZ \\
\hline \multirow[t]{4}{*}{ Congruent } & SD & Amplitude & $-2.248 \pm 0.600$ & $-0.416 \pm 0.487$ & $-0.403 \pm 0.505$ \\
\hline & & Latency & $408.142 \pm 3.027$ & $402.649 \pm 3.257$ & $401.855 \pm 3.580$ \\
\hline & $\mathrm{HC}$ & Amplitude & $-0.720 \pm 0.465$ & $0.792 \pm 0.652$ & $1.047 \pm 0.637$ \\
\hline & & Latency & $409.790 \pm 2.884$ & $405.945 \pm 2.695$ & $409.302 \pm 2.398$ \\
\hline \multirow[t]{4}{*}{ Incongruent } & SD & Amplitude & $-2.580 \pm 0.606$ & $-0.661 \pm 0.449$ & $-0.750 \pm 0.478$ \\
\hline & & Latency & $402.344 \pm 3.411$ & $402.100 \pm 2.946$ & $401.550 \pm 3.179$ \\
\hline & $\mathrm{HC}$ & Amplitude & $-0.185 \pm 0.464$ & $1.535 \pm 0.666$ & $1.695 \pm 0.702$ \\
\hline & & Latency & $411.621 \pm 2.902$ & $408.997 \pm 3.009$ & $412.171 \pm 2.838$ \\
\hline
\end{tabular}

$S P$

The amplitudes and latencies of SP were statistically analyzed. Please see Table 5 and Figure 4 for details. The ANOVA on SP amplitudes showed a main effect of electrode site $\triangle \mathrm{PZ}=1.523 \mu \mathrm{V} ; \mathrm{POZ}=-0.270 \mu \mathrm{V} ; \mathrm{OZ}=0.651 \mu \mathrm{V} ; \mathrm{F}(2,62)=25.201$, $\mathrm{P}<0.001)$; with the largest amplitudes in the $P Z$ and the smallest amplitudes in the POZ. There was a main effect of group $(H C=0.838 \mu V \otimes S u b D=0.431 \mu V ; F(1,31)=4.152, P=0.040<0.05)$, with smaller amplitudes in the SubD group than in the $\mathrm{HC}$ group. There was a main effect of word-face consistency (congruent=0.162 $\mu \mathrm{V}$; incongruent=1.107 $\mathrm{V} ; \mathrm{F}(1,31)=36.627, \mathrm{P}<0.001)$, and it was found that there was a trend interaction between group and word-face consistency $(F(1,31)=12.283, P<0.001)$, further analysis of the simple effect showed that the SubD group had a smaller differential wave of SP induced by discordant and consistent conditions than the $\mathrm{HC}$ group (Differential wave of $\mathrm{HC}$ group $=1.347 \mu \mathrm{V}$; Differential wave of SubD group=0.543 $\mu \mathrm{V}$; $\mathrm{P} \otimes$ 0.001).

The ANOVA on SP latencies showed all the effective effects are not significant.

\begin{tabular}{|c|c|c|c|c|c|}
\hline Task & Group & & $\overline{P Z}$ & $\overline{\mathrm{POZ}}$ & $\mathrm{OZ}$ \\
\hline \multirow[t]{4}{*}{ Congruent } & $\mathrm{SD}$ & Amplitude & $1.217 \pm 0.378$ & $-0.401 \pm 0.541$ & $-.336 \pm 0.396$ \\
\hline & & Latency & $748.840 \pm 5.440$ & $749.451 \pm 5.499$ & $755.249 \pm 5.160$ \\
\hline & $\mathrm{HC}$ & Amplitude & $-0.185 \pm 0.464$ & $1.047 \pm 0.637$ & $1.535 \pm 0.666$ \\
\hline & & Latency & $746.277 \pm 5.351$ & $744.385 \pm 4.867$ & $749.512 \pm 5.212$ \\
\hline \multirow[t]{4}{*}{ Incongruent } & SD & Amplitude & $-0.720 \pm 0.465$ & $1.695 \pm 0.702$ & $0.792 \pm 0.652$ \\
\hline & & Latency & $742.981 \pm 5.778$ & $754.211 \pm 5.637$ & $755.676 \pm 5.468$ \\
\hline & $\mathrm{HC}$ & Amplitude & $1.509 \pm .256$ & $1.043 \pm 0.220$ & $1.982 \pm 0.209$ \\
\hline & & Latency & $750.916 \pm 5.414$ & $735.901 \pm 4.898$ & $751.831 \pm 5.695$ \\
\hline
\end{tabular}

\section{Discussion}

Emotional conflict is a cognitive process in which we need to select our target information and suppress irrelevant information when processing emotional information, and the resolution of emotional conflict has great significance in real life. This study examined the behavioral and electrophysiological correlates of emotional conflict deficits in SubD patients.

The study found that subjects showed a significant behavioural Stroop interference effect. Both the SubD and HC groups had longer RTs in the incongruent condition than in the congruent condition when completing the emotional conflict task. This is consistent with the findings of Botvinic (Botvinick MM et al., 2004), who identified stronger activation in the prefrontal as well as the cingulate cortex in the incongruent condition, showing a significant decrease in behavioural indicators during response. Previous research (Etkin et al., 2006) has suggested that this is due to a conflict between emotional and cognitive resources. When word-face emotional valence is in conflict, word and face competition for cognitive resources, such as attention, and the resources taken up by processes such as recognition and processing of non-target stimuli can interfere with processes such as recognition and processing of target stimuli, and in turn make them less efficient. This study also found that 
the SubD group had significantly longer RT and lower ACC rates in both conditions compared to the HC group, suggesting that SubD may have deficits in the cognitive processes of emotional conflict.

Regarding the ERP data, the SubD group had greater N2 amplitude in the frontocentral region and greater N450 difference wave amplitude in the frontocentral region for "incongruent stimuli minus congruent stimuli" relative to the HC group. The SubD patients and HC group showed enhanced conflict SP for "incongruent stimuli" than for "congruent stimuli," and the HC group showed greater SP difference wave amplitude for "incongruent stimuli minus congruent stimuli" than the SubD patients. This, combined with the longer RT and lower ACC behavioural data during the task, suggests that SubD patients have neurological impairments in monitoring and responding to emotional conflict.

For N2, the subject type main effect was significant, showing a larger wave amplitude in the SubD group than in the HC group. N2 reflects the state of attention at the beginning of the entire cognitive processing, and the results suggest that SubD patients are more sensitive to the early processing of emotional stimuli than normal individuals. The present study did not find differences in the conflict condition, but previous studies found that the N2 amplitude of incongruent stimuli was greater than the N2 amplitude of congruent stimuli in the interference task (Gehring WJ et al., 1992; Kopp B et al., 1996; Holmes AJ et al., 2008), possibly because previous studies have mostly used the classical Stroop or go-nogo paradigms, whereas the present study used the word-face Stroop paradigm. Previous studies (Zhang JP et al., 2021) have shown that people with SubD pay different attention to emotional information than normal people and engage with emotional information to a greater extent, so the N2 amplitude was more negative in the SubD group, reflecting the fact that people with SubD require more cognitive resources to process emotional information.

For N450, the main effect of conflict type was significant, and waves in the incongruent condition had a greater turn in the frontal mid-lobe region than waves in the congruent condition. N450 is a valid indicator of the Stroop interference effect (Shen YM et al., 2013 ), and the findings suggest that there was a significant Stroop interference effect in this study. The main effect of subject type was significant, with the SubD group eliciting more negative N450 compared to the $\mathrm{HC}$ group, and an interaction effect between subject type and conflict type was found, with further simple effects analysis showing that the incongruent condition minus the more congruent condition elicited greater N450 in the SubD group than in the $\mathrm{HC}$ group.The $\mathrm{N} 450$ component can be used as an electrophysiological indicator of conflict processing efficiency (StrommerDavidovich $\mathrm{N}$ et al., 2018), which is positively correlated with the amount of psychological resources an individual devotes to conflict events (West R et al., 2005), and this study shows that people with SubD have low conflict processing efficiency and high interference sensitivity. It has also been suggested that N450 may be related to conflict monitoring (Liotti M et al., 2000; West R et al., 2003) and conflict resolution. For example, Qiu et al. (Qiu NJ et al., 2006) in a colour-word Stroop study using Chinese characters as materials found N450 responded to conflict monitoring and conflict resolution processes, so in the present study we suggest $\mathrm{N} 450$ reflects participants' monitoring and resolution of conflict between facial expressions and the meaning of emotional words associated with the facial word Stroop task. The greater wave of difference in N450 in the SubD group implies that SubD patients are more sensitive to the detection of emotional conflict.

For SP, a main effect of conflict type was significant, with incongruent conditions triggering greater SP than congruent conditions. Subject type main effects were evident, with the SubD group triggering smaller SP amplitudes than the HC group, which differed from the hypothesis, and an interaction effect between subject type and conflict type was found, with the SubD group inducing smaller waves of difference in SP in incongruent conditions minus congruent conditions than the HC group. Many studies (Whitney C et al., 2009; Clayson PE et al., 2011; Forster SE et al., 2011) have linked the slow wave of conflict SP to post-response monitoring and conflict adaptation. For example, Chen et al. (Chen A et al., 2011) suggest that conflict SP is related to post-response monitoring because this positivity extends beyond the mean response time. Larson et al. (M.J. Larson et al., 2009) suggest that post-response SP reflects the conflict adaptation process. Therefore, we suggest SP responds to subjects' monitoring of responses and conflict adaptation in the completion of an emotional conflict task. The results of the present study imply that adaptation and response monitoring are more delayed in people with SubD following emotional conflict resolution.

Research has shown that the inability to resolve and adapt to emotional conflict is typical of patients with certain mood disorders. The present study provides new behavioural and neurophysiological evidence for impaired emotional conflict control in patients with SubD. The findings suggest that, compared to the HC group, SubD patients have abnormal emotional conflict inhibition using the Word-Face Stroop task, as evidenced by greater sensitivity to early processing of emotional stimuli, overcommitment of cognitive resources, and sharper detection of emotional conflict, but delayed adaptation and response selection following emotional conflict. On the one hand, these findings help us to enhance our understanding of SubD inhibition defects, on the other hand, they also provide evidence on the cognitive neuropsychiatric model of SubD.

\section{Statement}

All subjects complete an informed consent form and agree to publication in an online open access publication.

The datasets generated during the current study are available in the [figshare] repository,

[https://figshare.com/articles/dataset/Dynamic_cognitive_characteristics_of_emotional_conflict_in_subthreshold_depressed_patients_An_Event-

Page $8 / 15$ 
The authors declare that they have no conflflicts of interest.

This study is not funded

Author contributions: Weizhou Wang performed the statistical analysis, wrote the manuscript, and was fully responsible for all content and editorial decisions. Qi Xie is responsible for the experimental design and implementation of the experiment. Weizhou Wang and Qi Xie contributed equally to the completion of the paper and are joint first authors of the article. Lulu Zhang is responsible for assisting in completing the experiment and collating the data. Associate Professor Shuang Hu designed the entire study, trained the experimentalists and critically evaluated the draft manuscript. All authors read and approved the final manuscript.

\section{References}

Algom, D., Chajut, E., \& Lev, S. (2004). A rational look at the emotional stroop phenomenon: a generic slowdown, not a stroop effect. Journal of experimental psychology: General, 133(3), 323.

Anderson, N. D., Lau, M. A., Segal, Z. V., \& Bishop, S. R. (2007). Mindfulness-based stress reduction and attentional control. Clinical Psychology \& Psychotherapy: An International Journal of Theory \& Practice, 14(6), 449-463.

Ballesteros, J., Bobes, J., Bulbena, A., Luque, A., Dal-Ré, R., Ibarra, N., \& Güemes, I. (2007). Sensitivity to change, discriminative performance, and cutoff criteria to define remission for embedded short scales of the Hamilton depression rating scale (HAMD). Journal of affective disorders, 102(1-3), 93-99.

Bertha, E. A., \& Balázs, J. (2013). Subthreshold depression in adolescence: a systematic review. European child \& adolescent psychiatry, 22(10), 589-603.

Botvinick, M. M., Cohen, J. D., \& Carter, C. S. (2004). Conflict monitoring and anterior cingulate cortex: an update. Trends in cognitive sciences, 8(12), 539-546.

Buntrock, C., Berking, M., Smit, F., Lehr, D., Nobis, S., Riper, H., ... \& Ebert, D. (2017). Preventing depression in adults with subthreshold depression: health-economic evaluation alongside a pragmatic randomized controlled trial of a web-based intervention. Journal of medical Internet research, 19(1), e5.

Chen, A., Bailey, K., Tiernan, B. N., \& West, R. (2011). Neural correlates of stimulus and response interference in a 2-1 mapping stroop task. International Journal of Psychophysiology, 80(2), 129-138.

Clayson, P. E., \& Larson, M. J. (2011). Conflict adaptation and sequential trial effects: Support for the conflict monitoring theory. Neuropsychologia, 49(7), 1953-1961.

Cuijpers, P., \& Smit, F. (2004). Subthreshold depression as a risk indicator for major depressive disorder: a systematic review of prospective studies. Acta psychiatrica scandinavica, 109(5), 325-331.

Dai, Q., \& Feng, Z. (2012). More excited for negative facial expressions in depression: evidence from an event-related potential study. Clinical neurophysiology, 123(11), 2172-2179.

De Pisapia, N., \& Braver, T. S. (2006). A model of dual control mechanisms through anterior cingulate and prefrontal cortex interactions. Neurocomputing, 69(10-12), 1322-1326.

Disner, S. G., Beevers, C. G., Haigh, E. A., \& Beck, A. T. (2011). Neural mechanisms of the cognitive model of depression. Nature Reviews Neuroscience, 12(8), 467-477.

Epp, A. M., Dobson, K. S., Dozois, D. J., \& Frewen, P. A. (2012). A systematic meta-analysis of the Stroop task in depression. Clinical psychology review, 32(4), 316-328.

Etkin, A., Egner, T., Peraza, D. M., Kandel, E. R., \& Hirsch, J. (2006). Resolving emotional conflict: a role for the rostral anterior cingulate cortex in modulating activity in the amygdala. Neuron, 51(6), 871-882.

Everaert, J., Koster, E. H., \& Derakshan, N. (2012). The combined cognitive bias hypothesis in depression. Clinical psychology review, 32(5), 413424. 
Ferrari, A. J., Charlson, F. J., Norman, R. E., Patten, S. B., Freedman, G., Murray, C. J., ... \& Whiteford, H. A. (2013). Burden of depressive disorders by country, sex, age, and year: findings from the global burden of disease study 2010. PLoS medicine, 10(11), e1001547.

Forster, S. E., Carter, C. S., Cohen, J. D., \& Cho, R. Y. (2011). Parametric manipulation of the conflict signal and control-state adaptation. Journal of Cognitive Neuroscience, 23(4), 923-935.

Gehring, W. J., Gratton, G., Coles, M. G., \& Donchin, E. (1992). Probability effects on stimulus evaluation and response processes. Journal of Experimental Psychology: Human Perception and Performance, 18(1), 198.

Hamilton, M. A. X. (1967). Development of a rating scale for primary depressive illness. British journal of social and clinical psychology, 6(4), 278-296.

Hayasaka, Y., Furukawa, T. A., Sozu, T., Imai, H., Kawakami, N., \& Horikoshi, M. (2015). Enthusiasm for homework and improvement of psychological distress in subthreshold depression during behavior therapy: secondary analysis of data from a randomized controlled trial. BMC psychiatry, 15(1), 1-11.

Holmes, A. J., \& Pizzagalli, D. A. (2008). Response conflict and frontocingulate dysfunction in unmedicated participants with major depression. Neuropsychologia, 46(12), 2904-2913.

Jiang, L., Wang, Y., Zhang, Y., Li, R., Wu, H., Li, C., .. \& Tao, Q. (2019). The reliability and validity of the center for epidemiologic studies depression scale (CES-D) for Chinese university students. Frontiers in psychiatry, 10, 315.

Jones, E. B., \& Sharpe, L. (2017). Cognitive bias modification: A review of meta-analyses. Journal of affective disorders, 223, $175-183$.

Kopp, B., Mattler, U., Goertz, R., \& Rist, F. (1996). N2, P3 and the lateralized readiness potential in a nogo task involving selective response priming. Electroencephalography and clinical Neurophysiology, 99(1), 19-27.

Kavcic, V., \& Clarke, J. M. (2000). Hemispheric interactions during a face-word Stroop-analog task. Neuropsychology, $14(4), 579$.

Larson, M. J., Kaufman, D. A., \& Perlstein, W. M. (2009). Neural time course of conflict adaptation effects on the Stroop task. Neuropsychologia, 47(3), 663-670.

LeDoux, J. E. (2000). Emotion circuits in the brain. Annual review of neuroscience, 23(1), 155-184.

Lee, Y. Y., Stockings, E. A., Harris, M. G., Doi, S. A. R., Page, I. S., Davidson, S. K., \& Barendregt, J. J. (2019). The risk of developing major depression among individuals with subthreshold depression: a systematic review and meta-analysis of longitudinal cohort studies. Psychological medicine, 49(1), 92-102.

Liotti, M., Woldorff, M. G., Perez III, R., \& Mayberg, H. S. (2000). An ERP study of the temporal course of the Stroop color-word interference effect. Neuropsychologia, 38(5), 701-711.

Lu, B., Hui, M. A., \& Yu-Xia, H. (2005). The Development of Native Chinese Affective Picture System-A pretest in 46 College Students. Chinese mental health journal.

Mikolajczyk, R. T., Maxwell, A. E., Naydenova, V., Meier, S., \& El Ansari, W. (2008). Depressive symptoms and perceived burdens related to being a student: Survey in three European countries. Clinical Practice and Epidemiology in Mental Health, 4(1), 1-9.

Ottowitz, W. E., Tondo, L., Dougherty, D. D., \& Savage, C. R. (2002). The neural network basis for abnormalities of attention and executive function in major depressive disorder: implications for application of the medical disease model to psychiatric disorders. Harvard review of psychiatry, 10(2), 86-99.

Qiu, J., Luo, Y., Wang, Q., Zhang, F., \& Zhang, Q. (2006). Brain mechanism of Stroop interference effect in Chinese characters. Brain Research, 1072(1), 186-193.

Radloff, L. S. (1977). A self-report depression scale for research in the general population. Applied psychol Measurements, 1, $385-401$.

Shen, Y., Xue, S., Wang, K., \& Qiu, J. (2013). Neural time course of emotional conflict control: an ERP study. Neuroscience letters, $541,34-38$.

Strommer-Davidovich, N., Lichtenstein-Vidne, L., Shrem, T., Alkoby, O., Richter-Levin, G., Rohr, C., \& Okon-Singer, H. (2018). F75. Selective Attention Processes in ADHD: Evidence From a Modified Stroop-Flanker Task. Biological Psychiatry, 83(9), S266-S267. 
Stenberg, G., Wiking, S., \& Dahl, M. (1998). Judging words at face value: Interference in a word processing task reveals automatic processing of affective facial expressions. Cognition \& Emotion, 12(6), 755-782.

Taake, I., Jaspers-Fayer, F., \& Liotti, M. (2009). Early frontal responses elicited by physical threat words in an emotional Stroop task: Modulation by anxiety sensitivity. Biological Psychology, 81(1), 48-57.

Thomas, S. J., Johnstone, S. J., \& Gonsalvez, C. J. (2007). Event-related potentials during an emotional Stroop task. International journal of psychophysiology, 63(3), 221-231.

Tipples, J., \& Sharma, D. (2000). Orienting to exogenous cues and attentional bias to affective pictures reflect separate processes. British Journal of Psychology, 91(1), 87-97.

Tuithof, M., Ten Have, M., van Dorsselaer, S., Kleinjan, M., Beekman, A., \& de Graaf, R. (2018). Course of subthreshold depression into a depressive disorder and its risk factors. Journal of affective disorders, 241, 206-215.

Van Hooff, J. C., Dietz, K. C., Sharma, D., \& Bowman, H. (2008). Neural correlates of intrusion of emotion words in a modified Stroop task. International journal of psychophysiology, 67(1), 23-34.

Williams, J. M. G., Mathews, A., \& MacLeod, C. (1996). The emotional Stroop task and psychopathology. Psychological bulletin, $120(1), 3$. Wang, Y., Huang, H., Yang, H., Xu, J., Mo, S., Lai, H., ... \& Zhang, J. (2019). Influence of EEG references on N170 component in human facial recognition. Frontiers in neuroscience, 13, 705.

Wei, D., Qiu, J., Tu, S., Tian, F., Su, Y., \& Luo, Y. (2010). Earthquake experience interference effects in a modified Stroop task: an ERP study. Neuroscience letters, 474(3), 121-125.

West, R., Jakubek, K., Wymbs, N., Perry, M., \& Moore, K. (2005). Neural correlates of conflict processing. Experimental brain research, 167(1), 3848.

West, R. (2003). Neural correlates of cognitive control and conflict detection in the Stroop and digit-location tasks. Neuropsychologia, 41(8), 1122-1135.

Whitney, C., Grossman, M., \& Kircher, T. T. (2009). The influence of multiple primes on bottom-up and top-down regulation during meaning retrieval: evidence for 2 distinct neural networks. Cerebral Cortex, 19(11), 2548-2560.

Xue, S., Wang, S., Kong, X., \& Qiu, J. (2017). Abnormal neural basis of emotional conflict control in treatment-resistant depression: An eventrelated potential study. Clinical EEG and neuroscience, 48(2), 103-110.

Zhang, J., Li, X., Du, J., Tan, X., Zhang, J., Zhang, Y., ... \& Kong, J. (2021). Impairments of Implicit Emotional Neurocognitive Processing in College Students With Subthreshold Depression: An ERP Study. Journal of Clinical Neurophysiology, 38(3), 192-197.

\section{Figures}




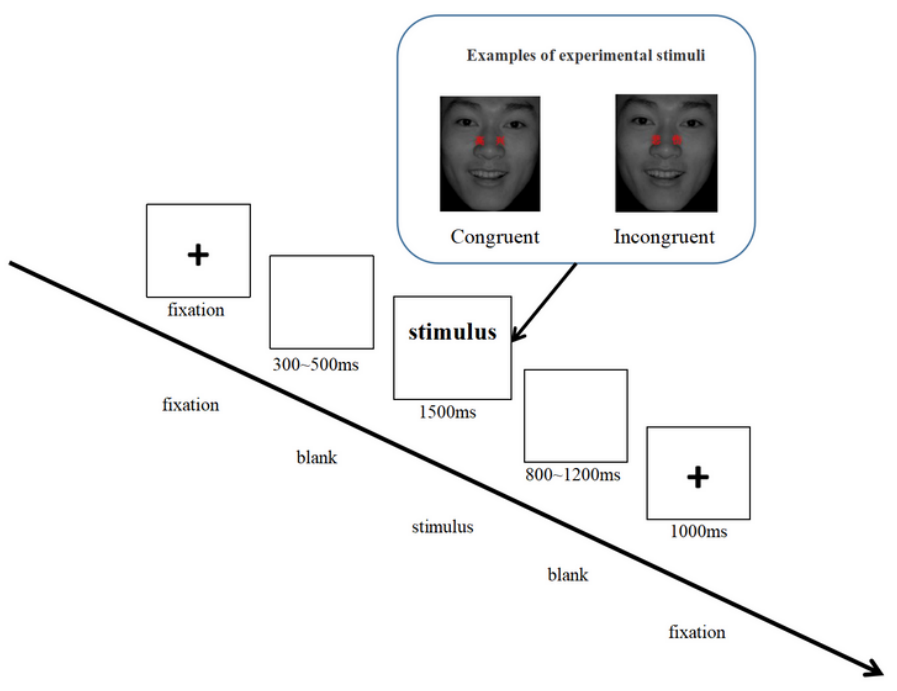

Figure 1. Illustration of one experimental trial in this study

\section{Figure 1}

See image above for figure legend. 


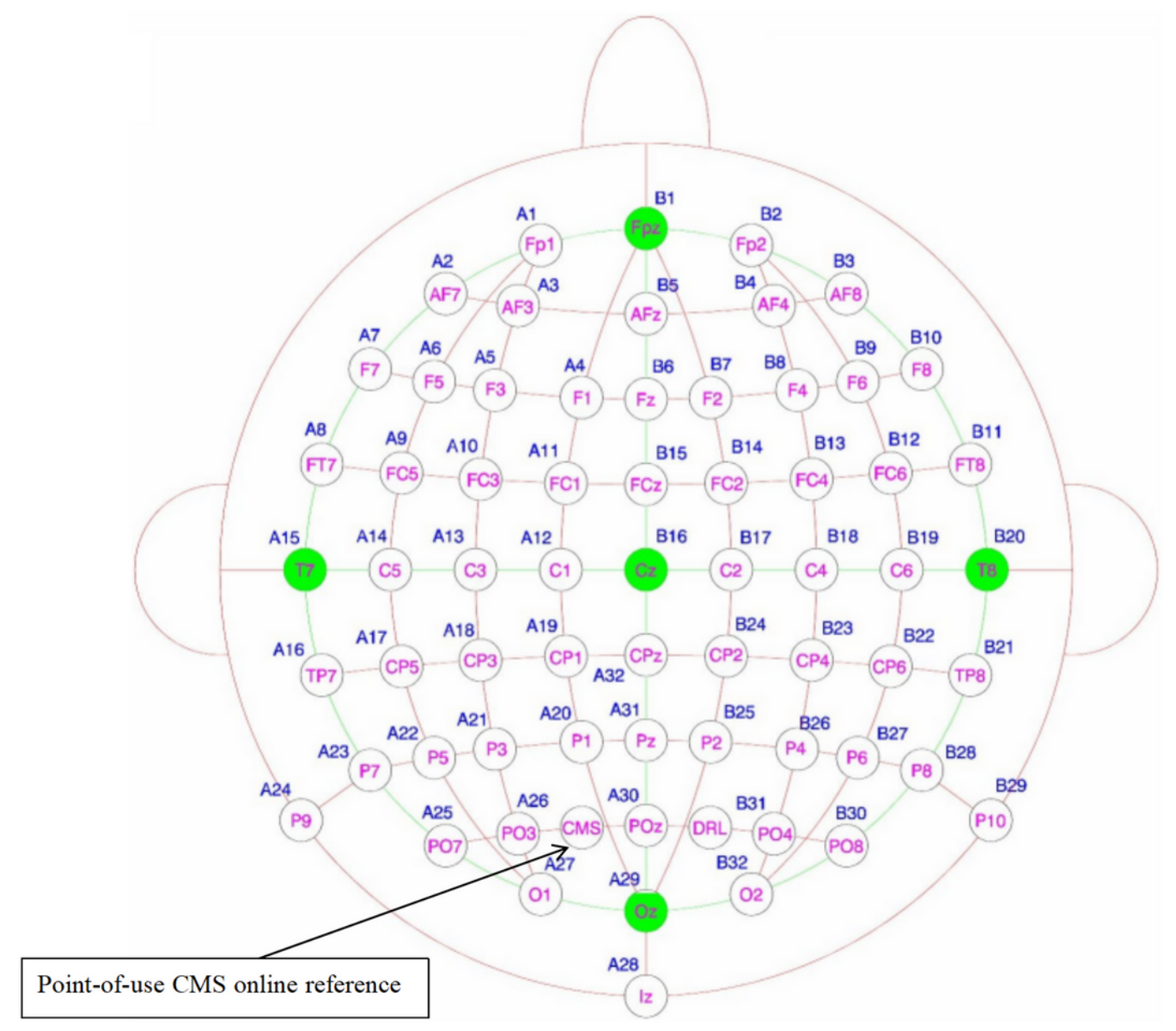

Figure 2. The 2D 64-sensor map

Figure 2

See image above for figure legend. 
A
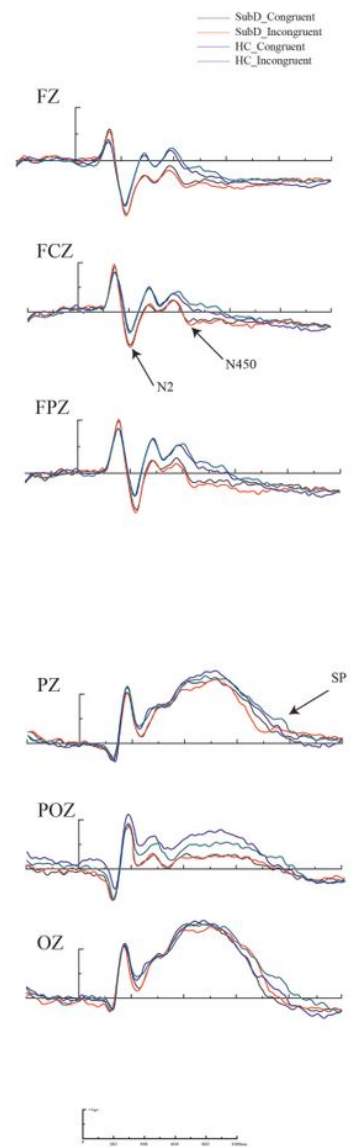

B

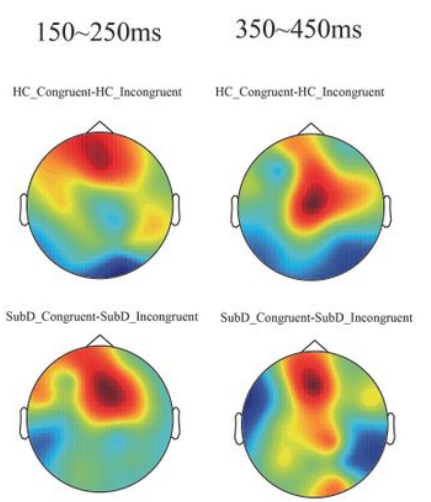

$700 \sim 800 \mathrm{~ms}$

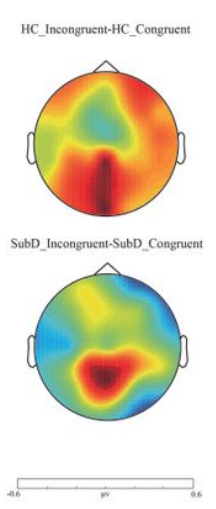

Figure 3.

A: Grand-average event-related potential (ERP) for emotional-congruent, and emotional-incongruent trials at FZ, FCZ, FPZ, Pz, POz and OZ.

B: The topographic map for different wave between incongruent trials minus congruent trials for SubD group and for HC group.

\section{Figure 3}

See image above for figure legend. 
A

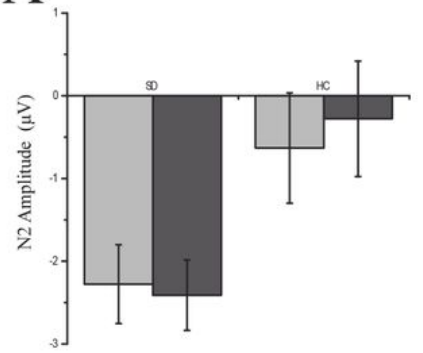

$\mathrm{C}$

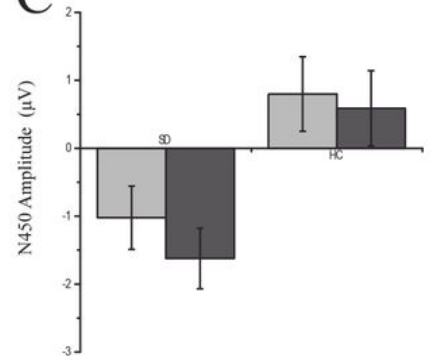

$\mathrm{E}$

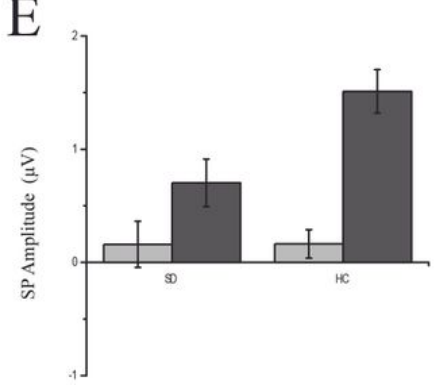

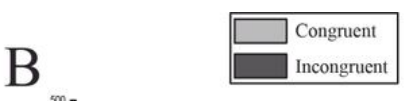

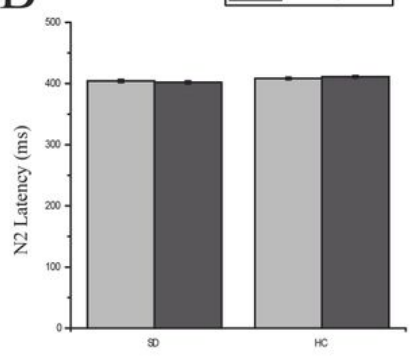

$\mathrm{D}$

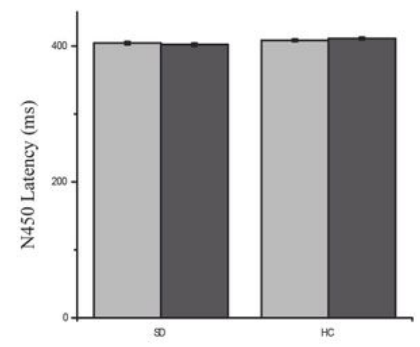

$\mathrm{F}$

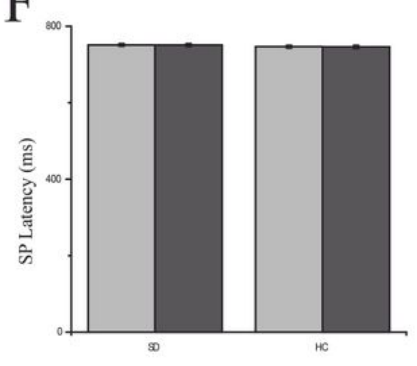

Figure 4. Mean amplitudes and latencies of N2, N450 and SP elicited by two stimuli in SD participants and HCs

\section{Figure 4}

See image above for figure legend. 